\title{
Pain, balance and mobility in people one year after total knee arthroplasty: A non-randomized cross- sectional pilot study contrasting posterior-stabilized and medial-pivot designs
}

Cathy Lo

Hong Kong Polytechnic University

Matthew Brodie

University of New South Wales

William Tsang

Open University of Hong Kong

Stephen Lord

University of New South Wales

Chun-hoi Yan

University of Hong Kong

Arnold Wong ( $\square$ arnold.wong@polyu.edu.hk)

https://orcid.org/0000-0002-5911-5756

\section{Research}

Keywords: Prosthesis, Postural sway, Gait, Wearable sensors, Post-operation, Balance performance

Posted Date: July 10th, 2020

DOI: https://doi.org/10.21203/rs.3.rs-26069/v2

License: (c) (i) This work is licensed under a Creative Commons Attribution 4.0 International License.

Read Full License 
Pain, balance and mobility in people one year after total knee arthroplasty: A pilot cross-sectional study contrasting posterior-stabilized and medialpivot designs

Authors:

Cathy WT Lo ${ }^{1}$, BSc, MPhil, cathy.wt.lo@ connect.polyu.hk

Matthew A Brodie 2 , PT, MSc, PhD,

matthew.brodie@neura.edu.au

William WN Tsang ${ }^{3}$, PT, MPhil, PhD, wntsang@ouhk.edu.hk

Stephen R Lord ${ }^{2}, \mathrm{PhD}$, s.lord@ neura.edu.au

Chun-Hoi Yan ${ }^{4}$, MBBS, FRCS, FRCSE, yanchunhoi@gmail.com

Arnold YL Wong ${ }^{1}$, PT, MPhil, PhD

Affiliations:

1. Department of Rehabilitation Sciences, The Hong Kong Polytechnic University, Hong Kong SAR, China

2. Neuroscience Research Australia, University of New South Wales, Australia

3. Department of Physiotherapy, School of Nursing and Health Studies, The Open University of Hong Kong, Hong Kong SAR, China

4. Department of Orthopaedics and Traumatology, The University of Hong Kong, Hong Kong SAR, China

Correspondence: $\quad$ Arnold YL Wong, PT, MPhil, PhD

Department of Rehabilitation Sciences

Hong Kong Polytechnic University

Hung Hom, Hong Kong, SAR, China

Phone: (+852) 2766-6741

Email: arnold.wong@polyu.edu.hk 


\begin{abstract}
Background: Total knee arthroplasty (TKA) is a common treatment for severe knee osteoarthritis. TKA designs have evolved from posterior-stabilized systems (PS-TKA) developed in the late 70s that replicate posterior-cruciate ligament function to medial-pivot systems (MP-TKA) developed in the early 90s that more closely mimic natural knee kinematics. This study aimed to test the hypothesis that people with MP-TKA have better postoperative outcomes than PS-TKA, and their balance control is more akin to age-matched asymptomatic controls.
\end{abstract}

Methods: Fifty-seven participants (20 PS-TKA, 18 MP-TKA and 19 asymptomatic controls) were recruited. At 1-year post-TKA, pain, knee stiffness and physical function were assessed using the Western Ontario and McMaster Universities Osteoarthritis Index (WOMAC). Static balance, mobility and gait stability were assessed using wearable devices during the near tandem stance, timed-up-and-go (TUG) and six-minute walk (6MWT) tests.

Results: Compared to asymptomatic controls, both TKA groups reported significantly more pain and stiffness, and demonstrated reduced functional mobility, increased stride-timevariability and impaired balance; indicating increased fall risk. After Bonferroni adjustment, no significant differences were observed between PS-TKA and MP-TKA participants. However, there was a trend for increased anteroposterior sway of the lumbar and head regions in the MP-TKA participants when undertaking the near tandem stance test.

Conclusions: Contrary to the hypothesis, the MP-TKA patients did not have less pain or better balance and mobility performance than the PS-TKA patients 1 year after surgery. 
Future longitudinal research should determine whether the two types of patients display different pain, balance controls and mobility over the first year postoperative period.

Keywords: Prosthesis; Postural sway; Gait; Wearable sensors; Post-operation; Balance performance

\section{Key messages regarding feasibility}

What uncertainties existed regarding the feasibility?;

- It is unclear whether patients with two common types of total knee arthroplasty (TKA) designs at 1-year follow-up would demonstrate different pain intensity, balance and mobility with reference to age-matched asymptomatic controls.

What are the key feasibility findings?

- Wearable sensors detected that both TKA groups reported significantly more pain and stiffness, and demonstrated reduced functional mobility, increased stride-timevariability, and poorer balance as compared to age-matched asymptomatic controls. However, there was a trend for increased anteroposterior sway of the lumbar and head regions in the medial-pivot systems (MP-TKA) participants when undertaking the near tandem stance test.

What are the implications of the feasibility findings for the design of the main study?

- Wearable sensors can be used in a clinical setting to measure balance and mobility of patients following TKA. A large-scale longitudinal study can be conducted to determine whether the two types of patients display significantly different pain, balance controls and mobility during the first year postoperative period. 


\section{Background}

Patients with knee osteoarthritis have impaired gait as indicated by spatio-temporal and kinematic gait deviations from healthy counterparts in level walking (1). Total knee arthroplasty (TKA) is a common procedure for treating patients with advanced and symptomatic knee osteoarthritis for pain relief and improved physical function (2). In response, TKA implants with differing "guided motion" systems have been designed to restore natural knee joint kinematics.

The posterior-stabilized (PS) knee system was developed in 1978 to mimic the posterior-cruciate ligament function (3). Specifically, the PS knee system uses a "cam-andpost" mechanism in which the mechanical role of the posterior-cruciate ligament is replaced by a post on the inlay and a cam on the femoral component $(4,5)$. The post and cam interact to prevent the anterior translation of the femur on the tibia while providing medial and lateral femoral posterior translation during knee flexion (4-6). Reported advantages include good soft tissue balancing, a large range of motion, predictable joint biomechanics, and the prevention of posterior tibial subluxation (7).

The medial-pivot (MP) knee system was developed in the early 90s based on the concept of medial centered rotation of a ball-and-socket joint as determined from knee kinematic studies (8-10). The natural knee kinematics in the MP knee system is replicated by using a more conforming surface in the tibial insert (11). This ultra-congruent medial compartment provides minimal anteroposterior motion, while the lateral compartment allows anteroposterior translation around a medial axis of rotation during knee flexion (11). 
Although patients with TKA display deficits in gait (e.g., range of motion or kinetics) and physical function as compared to age-matched asymptomatic controls, it remains unclear if the deficits are prosthesis-related (12-14). Without such information, it is difficult for clinicians to make clinical decisions with respect to the appropriate knee implant for their patients. Different TKA designs should theoretically alter knee kinematics and associated balance and mobility performance (12). A recent in vitro study using ultrasonic 3dimenstional motion analysis observed significant differences in tibiofemoral kinematics during simulated deep knee flexion from $20^{\circ}$ to $120^{\circ}$ of flexion between PS- and MP-TKA implanted human knee specimens (5). However, Bae et al. found no significant differences in clinical outcomes (including self-reported pain and physical function, knee range of motion, femorotibial angle, patella tilt angle and the postoperative patellar translation) between the PS- and MP-TKAs at a post-operative 5-year follow-up study (15).

While optoelectronic capturing systems, instrumented treadmills and force platforms are traditionally used for gait analysis following TKA $(12,16,17)$, wearable inertial sensors have been increasingly used as an inexpensive means to quantify gait in a non-laboratory environment (18). Since wearable devices can measure the three-dimensional acceleration of body parts and estimate velocity, displacement and gait stability and variability (18-20), and can characterize altered pattern in patients with TKA during functional tasks. Data from wearable devices attached to the head and pelvis have been validated with respect to detecting stride-to-stride oscillations during walking (20), for characterizing patients with Parkinson's disease and older people at risk of falls (19-21).

Given the above, this study aimed to compare: (a) static and dynamic balance, gait stability and physical performance using wearable sensors between people who received PS- 
TKA and MP-TKA one year after surgery; and (b) balance and mobility of these patients and age-matched asymptomatic controls. Since MP-TKA implants are characterized by a highly congruent medial tibiofemoral articulation, which may better replicate the natural kinematics of the knee, we hypothesized that 1) people who received MP-TKA would report less pain and perform better in the balance and mobility tests than those with PS-TKA; 2) the test performances in the MP-TKA group would be more akin to those found in age-matched asymptomatic controls.

\section{Methods}

\section{Participants}

Patients with PS- and MP-TKA were recruited to the current cross-sectional study from the division of Joint Replacement Surgery from a local Hospital. Potential TKA participants were recruited if they met the inclusion criteria: 60 years or older, independent walking for at least 10 minutes indoors without using a walking aid, no contraindication to exercises. Participants were excluded if they had a diagnosis of neurological or vestibular impairment, uncontrolled cardiopulmonary disorders, severe diabetes mellitus, rheumatic arthritis, a body mass index $(\mathrm{BMI}) \geq 40 \mathrm{~kg} / \mathrm{m}^{2}$, a knee flexion contracture $\geq 10^{\circ}$, or Kellgren and Lawrence grade $\geq 3$ knee osteoarthritis on the non-operated knee indicating absence of moderate or severe arthritis, a history of lower extremity fracture or surgery other than the primary unilateral TKA, and recent lower extremity musculoskeletal injuries that precluded an individual from participating in the study. These patients were excluded because their medical conditions might affect physical performance and confound our findings.

Furthermore, patients were excluded if they had major postoperative complications such as superficial or deep infections, deep venous thrombosis, pulmonary embolism or wound healing problems. Patients with unilateral TKA with either Attune Posterior Stabilized Knee 
Prosthesis (DePuy Orthopaedics Inc., Warsaw, USA) or Evolution Medial Pivot Knee Prosthesis (Wright Medical Technology, Arlington, Tennessee, USA) were eligible.

TKAs in both groups were implanted by two senior orthopaedics surgeons (one is the co-author CHY) who have been performing both designs (MP \& PS prostheses) for more than 5 years. Standard surgical techniques included a longitudinal skin incision, medial parapatellar approach, subperiosteal dissection over the medial tibial plateau, and resurfacing of the patella depending on the intraoperative wear pattern. Surgery was undertaken under spinal or combined spinal and epidural anesthesia and lasted for 90 to 120 minutes. Patients received standard, weight-based doses of preoperative antibiotics and intravenous tranexamic acid, followed by 2 weeks of venous thromboembolism prophylaxis with aspirin. Inpatient physiotherapy was commenced on postoperative day 0 under the management of physiotherapists in order to achieve full weight bearing on the operated leg. Active, activeassisted, and passive knee mobilization exercises were progressively prescribed. Patients were trained to walk with a frame or a quadripod depending on their progress. Additionally, they were given an exercise pamphlet regarding lower limb stretching and mobilization exercises to perform at home following discharge.

Age-matched asymptomatic controls comprised community senior center attendees. Inclusion criteria for asymptomatic controls included: no signs of stiffness or pain in the knees in the last year; and able to walk unaided both indoors and outdoors. Exclusion criteria for asymptomatic controls followed those of participants with TKA. The study was approved by the Human Subjects Ethics Sub-committee of the University. All participants provided informed consent before participation. 


\section{Self-reported pain, stiffness and physical functioning}

Pain, stiffness and physical functioning of all participants were measured with the Chinese version of the Western Ontario and McMaster Universities Osteoarthritis Index (WOMAC) (22). This activity-based self-administered questionnaire comprises 24 questions related to knee pain (5 items), knee stiffness ( 2 items) and physical functions (17 items). WOMAC has demonstrated good test-retest reliability for evaluating the TKA population (intra-class correlation coefficient $=0.82,0.88,0.84$ for pain, stiffness and function respectively) (22). A higher score in each subscale indicates that the respondent has more knee pain, more knee stiffness or poorer physical function.

\section{Mobility assessments}

Mobility was assessed with two performance-based tests: the timed-up-and-go test (TUG) and the six-minute walk test (6MWT). These tests are commonly used to evaluate the functional recovery after TKA $(23,24)$. The TUG has an excellent reported inter-rater reliability with an intraclass correlation coefficient of 0.99 , and is a functional test of strength, agility and dynamic balance (25). Participants were instructed to rise from an armless chair (seat height of $46 \mathrm{~cm}$ ), walk unaided at a self-selected comfortable pace along a line on the floor for 3 meters, and then turn and walk back to the chair and sit down (25). Participants performed a practice trial, followed by two experimental trials with the faster trial time used for analysis. The 6MWT assesses walking endurance/tolerance and has demonstrated excellent inter-rater reliability (intraclass correlation coefficient of 0.91) (26). The test was demonstrated and then participants were asked to walk as quickly as possible back and forth along a 20m hallway for 6 minutes without running or jogging (27). Participants were allowed to slow down, to stop, or to rest, if necessary. A standard set of 
recommended instructions and encouraging statements were used (27). The total distance covered was recorded for analysis.

\section{Standing balance and gait stability assessments}

Balance parameters during a standing task and during the 6MWT were assessed with two synchronized inertial sensors (Figure 1). Each sensor contained a tri-axial accelerometer and a tri-axial gyroscope (Opal, APDM Inc, Portland, OR, USA; sampling frequency 128 $\mathrm{Hz}$ ). The lumbar sensor was firmly strapped onto the participant with a belt approximately at the L5 level (near the body center of mass). The head sensor was attached to a plastic helmet that was secured at the vertex of the participant's head. The three-dimensional angular velocity and acceleration data from each sensor were collected during both static and dynamic tasks and processed using a custom-written program (MATLAB, Natick, MA, USA).

$\langle<<<<$ Please insert Figure 1 here $\gg>>>>$

Postural sway was assessed using the near-tandem stance test with eyes open. Participants were instructed to fold their arms across the chest and to stand with the heel of front foot (dominant foot: determined by self-reported leg dominance using the question of which leg will be used to shoot a ball on a target regardless of any pain on lower limbs) $2.5 \mathrm{~cm}$ anterior and $2.5 \mathrm{~cm}$ lateral (marked by a $2.5 \mathrm{~cm}$ x $2.5 \mathrm{~cm}$ cardboard template) to the great toe of the rear foot on a hard surface for 30 seconds. The head and lumbar static balance parameters were assessed over the middle 25 seconds to prevent any movements at the beginning or end of the test affecting the results. Static balance parameters included the $95 \%$ range of sway in degrees $(\Theta)$ in the anteroposterior (AP) and mediolateral (ML) directions 
called pitch and roll, respectively (Equations $1 \& 2$ ). The root mean square (RMS) of angular velocity was calculated by combining movements about all axes into one parameter (Figure 2), where $\mathrm{fA}$ is the acceleration of the sensor after low-pass filtering with a $4^{\text {th }}$ order bidirectional Butterworth filter at a cut-off frequency of one hertz.

$$
\begin{aligned}
& \theta_{A P(\text { Pitch })}=\sin ^{-1} \frac{f A_{A P}}{\sqrt{f A_{A P}^{2}+f A_{M L}^{2}+f A_{V T}^{2}}} \text { Equation } 1 \\
& \theta_{M L(\text { Roll })}=\sin ^{-1} \frac{f A_{M L}}{\sqrt{f A_{A P}^{2}+f A_{M L}^{2}+f A_{V T}^{2}}} \text { Equation } 2
\end{aligned}
$$

Note: VT-vertical

$$
<<<<<\text { Please insert Figure } 2 \text { here } \gg>>>>
$$

Dynamic balance was assessed during the periods of straight line walking in the 6MWT from multiple 20-meter laps (Figure 3). Turns were identified and excluded from the analysis by the gyroscope threshold of $30 \%$ s about the vertical axis. To prevent false identification of transient gait rotations instead of turns the gyroscope data were low-pass filtered using a $4^{\text {th }}$ order bidirectional Butterworth filter with a 0.5 hertz cut-off frequency and a total turn rotation of at least $90^{\circ}$ was required (Figure 4). For each gait parameter the robust mean was calculated (mean after excluding the best lap and worst lap). Steps during each straight line lap were identified by heel strike acceleration peaks (28). Cadence was calculated as the number steps per minute. Stride time variability was the standard deviation of consecutive strides ( 1 stride $=2$ steps $)$; greater variability has been associated with increased fall risk (29). Step time asymmetry was calculated as the absolute difference between left and right step times as a percentage (28). Relative displacements of the head and lumbar were reported as the RMS along the AP, ML and VT and calculated from the sensor 
data using validated methods (30); reduced VT displacements correlate with less vigorous gait while increase transverse plane displacements are associated with reduced gait stability (19). Finally, harmonic ratios (HR) were used as a measure of rhythm/smoothness of head and lumbar accelerations during walking. Lower HRs indicate reduced dynamic balance and are associated with increased fall risk $(31,32)$.

$\ll<<<<$ Please insert Figure 3 here $\gg \gg>>>$

$\ll<<<$ Please insert Figure 4 here $\gg>>>>$

\section{Statistical analysis}

All statistical analyses were performed using SPSS software (Version 22, IBM Corp., Armonk, NY). Non-parametric tests were used for the analysis as most variables did not meet the requirements for normality as determined by Shapiro-Wilks tests of normality.

Demographic variables specific to participants with TKA (e.g., months after operation and the percentage of participants using walking aids outdoor) were compared between the PSTKA and MP-TKA participants using Mann-Whitney U tests (for continuous variables) or Chi-square test (for nominal variables). For the remainder of the demographic and clinical variables of interest, Kruskal-Wallis tests (for continuous variables) and chi-square tests (for nominal variables) were used to compare the differences among PS-TKA, MP-TKA and asymptomatic controls. The significance level was set at 0.05 (2-tailed) and post-hoc analysis using Bonferroni adjustments were performed. Effect sizes $(r)$ of each observed difference were calculated by dividing the $Z$ value by the square root of the total number of participants in that pair of groups (33). Cohen's guidelines for $r$ suggest that small, medium and large effect sizes are $0.1,0.3$ and 0.5 respectively (34). 


\section{Results}

\section{Participants}

Demographic data of the 57 participants (20 PS-TKA, 18 MP-TKA and 19 controls) are shown in Table 1. There were no significant age and gender differences among the groups. The two TKA groups showed no significant differences in post-surgery duration, number of falls or trips within 1 year before surgery, number of falls or trips before and after operation, and the proportions using an assistive device outdoors. The control group participants took significantly fewer medications and had lower BMIs, whereas the two TKA groups did not differ significantly with respect to these parameters. There were no significant differences in the number of falls or trips in the previous 12 months among the three groups.

$\ll<<<<$ Please insert Table 1 here $\gg>>>>$

\section{Pain, stiffness and physical functioning}

No significant differences were detected in self-reported knee pain, knee stiffness and physical activity limitations between the two TKA groups. However, both PS-TKA and MPTKA participants perceived significantly more knee pain, knee stiffness and physical activity limitations than the asymptomatic controls (Table 2).

\footnotetext{
$\ll<<<<$ Please insert Table 2 here $>>>>>$
}

\section{Mobility assessments}

No significant difference in 6MWT distance was noted between the two TKA groups. Compared to the asymptomatic controls, both TKA groups had significantly shorter 6MWT 
walking distances. There was a trend indicating the MP-TKA group had slower TUG times than the control group (Table 2).

\section{Postural sway in the near-tandem stance test}

In the near-tandem stance test, significant differences in sway in the AP direction assessed the level of the head and lumbar regions were observed among the three groups (Table 3). Post-hoc analyses showed that the MP-TKA participants has greater head and lumbar AP sway than the asymptomatic controls, but that these sway measures did not differ between the two TKA groups. The absolute values for sway were generally greater in the ML axis compared with the AP axis in all three groups (except the lumber sway of MP-TKA).

$\ll<<<$ Please insert Table 3 here $\gg \gg \gg>$

\section{Gait speed and stability}

Signicant differences were observed for cadence, stride time variabilty and step time asymmetry among the three groups (Table 3). Post-hoc analyses revealed that MP-TKA participants demonstrated significantly greater stride time variability and step time asymmetry than asymptomatic controls, while PS-TKA participants had significantly reduced cadence and greater stride time variability than asymptomatic controls. The two TKA groups did not differ significantly in any gait parameters.

Regarding oscillatory displacements during the 6MWT, significant differences were observed in the ML and VT directions (Figure 3, Table 3). Compared to asymptomatic controls, the MP-TKA participants had significantly greater ML oscillatory displacements and significantly smaller VT oscillatory displacements assessed at both the head and lumbar 
levels. For the PS-TKA participants, these differences were only detectable at the level of the head (Table 3).

The three groups demonstrated significantly different HRs all the three axes at both the head and lumbar levels during the 6MWT (Table 3). Post-hoc tests revealed that the MPTKA participants had significantly lower head and lumbar HRs than asymptomatic controls in 5 of the 6 parameters, while the PS-TKA participants only had significantly lower head HRs in AP axis than the asymptomatic controls. No significant difference in HRs were evident between the two TKA groups.

\section{Discussion}

This study compared pain, balance, gait stability and physical performance in people who received a PS-TKA and a MP-TKA one year after surgery, as well as between these two groups and age-matched asymptomatic controls. Since the two TKA designs adopt different mechanisms to restore natural knee kinematics, we hypothesized that people with a MP-TKA (because of its highly congruent medial tibiofemoral articulation) would have superior outcomes to those with a PS-TKA, and their physical function would be more in line with that observed in asymptomatic controls. Contrary to our hypothesis, there were no significant differences in pain, balance and mobility outcomes between the two TKA groups at one-year post-surgery. Both TKA groups reported more pain, demonstrated inferior physical performances (e.g. shorter 6MWT distances), increased postural sway in a challenging stance position and poorer gait. Specifically, both TKA groups had increased stride-time-variability and reduced harmonic ratios, which together indicate gait instability and a potential higher risk of falls. 
The TKA designs may play a role in dynamic trunk control during walking. Recent research has shown that the knee flexes from $0^{\circ}$ to $45^{\circ}$ during stance phase, which involves a lateral-pivot kinematic pattern of the knee (from heel strike to flat foot). This is followed by the medial-pivot pattern in later flexion range (from flat foot to push off) $(35,36)$. The medial-pivot kinematics adopted in the MP-TKA may limit the natural lateral-pivot pattern in the early flexion during walking due to the lack of lateral constraint combined with a single radius of curvature femoral component design (instead of a traditional multi-radius design) and more normal tensioning of the collateral ligaments (37). Conversely, since the PS-TKA prosthesis allows mediolateral posterior translation during flexion (5), the lateral-pivot pattern is facilitated by PS-TKA. Hence, PS-TKA patients may be expected to have better step time symmetry and better control of lateral oscillations during gait as compared to MPTKA patients.

The lack of significant differences in the WOMAC pain, stiffness and functioning outcomes between the PS-TKA and MP-TKA concurs with previous research that has reported no significant differences in self-reported clinical outcomes between PS-TKA and MP-TKA patients 5 to 6 years after TKA $(15,38)$. Further, Warth et al. observed that the presence of an intraoperative medial-pivot kinematic pattern of TKA implants was not necessarily associated with better subjective outcomes at one year post-surgery (39). The poorer self-reported functional status of both PS-TKA and MP-TKA participants as compared to asymptomatic controls in the current study is consistent with prior studies (12, 40), although Yoshida et al. found that self-reported functional status in TKA patients was equivalent to asymptomatic controls at 12 months post-surgery (24). 
The TKA groups showed comparable TUG performances, but poorer 6MWT performances compared with asymptomatic controls. The shorter 6MWT distances in TKA groups is in line with two previous studies conducted in people with unspecified TKA of similar ages $(23,40)$. However, Yoshida et al. found no significant differences in TUG and 6MWT results between post-TKA participants and asymptomatic controls at one year after TKA (24). Interestingly, the average performance of TKA participants in the abovementioned studies by Bade et al. (TUG: 9.1s; 6MWT: 432.6m), Ko et al. (TUG: 7.4s; 6MWT: 432.5m) and Yoshida et al. (TUG: 6.8s; 6MWT: 630.0m) were better than that of our TKA participants (mean TUG: 15.5s; mean 6MWT: 301.7m) and asymptomatic controls (mean TUG: 12.0s; mean 6MWT: 403.7m). These differences might be due to our TKA participants not undergoing any structured post-operative rehabilitation programs, resulting in lower physical activity levels (41). Both TKA groups took more medications and had increased BMI compared to the control group. This suggests that the decreased mobility (6MWT) and increased pain (WOMAC) in the TKA participants might have negatively influenced their health outcomes. Future prospective studies should investigate whether post-operative rehabilitation could help close these substantial gaps.

With respect to standing balance, AP sway measured at the head and lumbar regions differed between the two TKA groups and the asymptomatic controls, whereas no such differences were evident for ML sway. Previous research has reported that participants place approximately $70 \%$ of their body weight on the rear foot during the tandem stance (42), while the AP rhythmic weight shifting plays a functional role in maintaining lateral stability (47). Increased AP sway in MP-TKA patients may indicate they are less able to detect or control their sway in a challenging balancing stance or they need to transfer weight between the two feet to maintain their lateral stability. The trend of poorer standing balance in MP-TKA 
participants (Figure 2) runs counter to our hypothesis, in which MP-TKA participants would have superior balance control than PS-TKA counterparts.

There were no significant differences in stride-to-stride oscillations and HR gait parameters between the two TKA groups (Table 3), but cadence was decreased, VT displacement was reduced, and ML displacement, stride time variability and step time asymmetry were increased in both TKA groups compared with the asymptomatic controls (Figure 3). Furthermore, the two TKA groups demonstrated reduced HRs indicating less smooth and more unstable walking patterns $(48,49)$. Collectively, these results indicate both TKA groups had suboptimal dynamic stability and increased risk of falls even at one year after surgery.

The limitations of this study include: first, mild knee osteoarthritis in the non-operated knee of some TKA participants might have affected their pain, balance and mobility. Second, unmeasured physical factors (e.g., impaired vision, proprioception and muscle strength) might have influenced our findings although people with diagnosed visual and proprioception impairments were excluded. Third, multiple outcomes were measured given the exploratory nature of this study; factors that might have resulted in both type 1 and type 2 errors. Further, the sample size was relatively small and thus might have been underpowered with respect to some outcome measures. However, both self-reported and objective measures consistently indicated that there was no detectable difference in outcomes between PS-TKA and MP-TKA participants, while TKA participants demonstrated significantly poorer balance and mobility than age-matched asymptomatic controls. Fourth, since participants were not randomized into different TKA or control groups nor assessed for any pre-surgical outcomes, it is not possible to rule out pre-exiting differences in health and functional abilities between the two TKA 
groups and the control group. Both TKA groups took more medications and had higher BMI values, which implied poorer health outcomes. In addition, preoperative physical function and balance control measures were not collected. Given the cross-sectional design of this study, it is not possible to determine if increased pain and reduced functional abilities contributed to the increased BMI or vice versa. A prospective study with a larger sample as well as pre- and post-assessments at different time points is warranted to clarify the effects of different TKA prosthesis designs on the changes of health outcomes, postoperative balance and physical function during the first-year post operation. Finally, level ground walking in the current study may be insensitive to detect differences in functional performance between the two TKA groups because the medial-pivot pattern only occurs at knee flexion beyond $45^{\circ}$ in natural knee kinematics (35). Future research should include additional functional tasks (e.g. stair ascent and descent, single-leg testing) to detect subtle differences in functional performance/ balance control between PS-TKA and MP-TKA patients.

In summary, contrary to our hypothesis, the MP-TKA group did not report less pain or perform better than the PS-TKA group in a range of balance and mobility tests one year after surgery. Future studies should investigate whether postoperative rehabilitation programs can improve pain, balance and mobility outcomes so that those with TKA can stand and mobilize similarly to asymptomatic individuals. 


\section{List of abbreviations}

\begin{tabular}{|l|l|}
\hline 6 MWT & Six-minute walk test \\
\hline AP & Anteroposterior \\
\hline BMI & Body mass index \\
\hline HR & Harmonic ratios \\
\hline ML & Mediolateral \\
\hline MP & Medial-pivot \\
\hline PS & Posterior-stabilized \\
\hline RMS & Root mean square \\
\hline TKA & Total knee arthroplasty \\
\hline TUG & Timed-up-and-go \\
\hline VT & Vertical \\
\hline WOMAC & Western Ontario and McMaster Universities Osteoarthritis Index \\
\hline
\end{tabular}

\section{Declarations}

\section{Ethics approval and consent to participate}

Ethical approval was obtained from the Human Subjects Ethics Sub-committee of the

University and local hospitals. All participants provided informed consent before participation.

\section{Consent for publication}

The authors agree to the content for publication.

\section{Availability of data and materials}

The datasets generated and/or analyzed during the current study are not publicly available due to personal data confidentiality policy but are available from the corresponding author on reasonable request.

\section{Competing interests}


The authors declared no potential conflicts of interest with respect to the research, authorship, and/or publication of this article.

\section{Funding}

This work was supported by The Hong Kong Polytechnic University Start-up fund (grant number 1-ZE4G).

\section{Authors' contributions}

CL \& AW were responsible for the conception and design of the research, reviewing articles, analyzing data, interpreting the results, writing and drafting the manuscript, and revision of the article for important intellectual content. WT, MB \& SL were responsible for interpreting results of the research and revising the manuscript. $\mathrm{CHY}$ was responsible for the revision of the article for important intellectual content. All authors read and approved the final version of the manuscript.

\section{Acknowledgements}

The authors would like to thank all participants Cyrus YC Ip, Clement CY Leung, Alan CL Tam and Allen WH Wong in data collection in the current study. 


\section{References}

1. Mills K, Hunt MA, Ferber R. Biomechanical Deviations During Level Walking Associated With Knee Osteoarthritis: A Systematic Review and Meta-Analysis. Arthritis Care Res (Hoboken). 2013;65(10):1643-65. doi:10.1002/acr.22015

2. Felson DT, Lawrence RC, Dieppe PA, Hirsch R, Helmick CG, Jordan JM, et al. Osteoarthritis: new insights. Part 1: the disease and its risk factors. Ann Intern Med. 2000;133(8):635. doi:10.7326/0003-4819-133-8-200010170-00016

3. Dorr LD, Ochsner JL, Gronley J, Perry J. Functional comparison of posterior cruciate-retained versus cruciate-sacrificed total knee arthroplasty. Clin Orthop Relat Res. 1988(236):36.

4. Blevins JL, Cross MB. Prosthetic Kinematics: Cruciate Retaining Versus Posterior Stabilized Versus Medial Pivot. In: Rodríguez-Merchán EC, Oussedik S, editors. Total Knee Arthroplasty: A Comprehensive Guide. Cham: Springer International Publishing; 2015. p. 137-43.

5. Steinbrück A, Schröder C, Woiczinski M, Fottner A, Pinskerova V, Müller PE, et al. Femorotibial kinematics and load patterns after total knee arthroplasty: An in vitro comparison of posterior-stabilized versus medial-stabilized design. Clin Biomech. 2016;33:42-8. doi:10.1016/j.clinbiomech.2016.02.002

6. Kolisek FR, McGrath MS, Marker DR, Jessup N, Seyler TM, Mont MA, et al. Posterior-stabilized versus posterior cruciate ligament-retaining total knee arthroplasty. The Iowa Orthop J. 2009;29:23-7.

7. Insall JN, Lachiewicz PF, Burstein AH. The posterior stabilized condylar prosthesis: a modification of the total condylar design. Two to four-year clinical experience. J Bone Joint Surg Am. 1982;64(9):1317-23.

8. Bae DK, Song SJ, Cho SD. Clinical Outcome of Total Knee Arthroplasty With Medial Pivot Prosthesis: A Comparative Study Between the Cruciate Retaining and Sacrificing. J Arthroplasty. 2011;26(5):693-8. doi:10.1016/j.arth.2010.04.022

9. Blaha JD, Mancinelli CA, Simons WH, Kish VL, Thyagarajan G. Kinematics of the human knee using an open chain cadaver model. Clin Orthop Relat Res. 2003(410):25.

10. Iwaki H, Pinskerova V, Freeman MA. Tibiofemoral movement 1: the shapes and relative movements of the femur and tibia in the unloaded cadaver knee. J Bone Joint Surg Br. 2000;82(8):1189. doi:10.1302/0301-620X.82B8.0821189

11. Shimmin A, Martinez-Martos S, Owens J, Iorgulescu AD, Banks S. Fluoroscopic motion study confirming the stability of a medial pivot design total knee arthroplasty. The Knee. 2015;22(6):522-6. doi:10.1016/j.knee.2014.11.011

12. McClelland JA, Webster KE, Feller JA. Gait analysis of patients following total knee replacement: A systematic review. The Knee. 2007;14(4):253-63.

doi:10.1016/j.knee.2007.04.003

13. Meneghini RM, Deckard ER, Ishmael MK, Ziemba-Davis M. A Dual-Pivot Pattern Simulating Native Knee Kinematics Optimizes Functional Outcomes After Total Knee Arthroplasty. J Arthroplasty. 2017;32(10):3009-15. doi:10.1016/j.arth.2017.04.050 14. Noble PC, Gordon MJ, Weiss JM, Reddix RN, Conditt MA, Mathis KB. Does total knee replacement restore normal knee function? Clin Orthop Relat Res. 2005(431):157. 15. Bae DK, Cho SD, Im SK, Song SJ. Comparison of Midterm Clinical and Radiographic Results Between Total Knee Arthroplasties Using Medial Pivot and PosteriorStabilized Prosthesis-A Matched Pair Analysis. J Arthroplasty. 2016;31(2):419-24. doi:10.1016/j.arth.2015.09.038

16. Caldas R, Mundt M, Potthast W, Buarque de Lima Neto F, Markert B. A systematic review of gait analysis methods based on inertial sensors and adaptive algorithms. Gait Posture. 2017;57:204-10. doi:10.1016/j.gaitpost.2017.06.019 
17. Tesio L, Rota V, Chessa C, Perucca L. The 3D path of body centre of mass during adult human walking on force treadmill. J Biomech. 2010;43(5):938-44.

doi:10.1016/j.jbiomech.2009.10.049

18. Kavanagh JJ, Menz HB. Accelerometry: A technique for quantifying movement patterns during walking. Gait Posture. 2008;28(1):1-15. doi:10.1016/j.gaitpost.2007.10.010 19. Brodie MA, Canning CG, Beijer TR, Lord SR. Uncontrolled head oscillations in people with Parkinson's disease may reflect an inability to respond to perturbations while walking. Physiol Meas. 2015;36(5):873-81. doi:10.1088/0967-3334/36/5/873

20. Brodie MAD, Beijer TR, Canning CG, Lord SR. Head and pelvis stride-to-stride oscillations in gait: validation and interpretation of measurements from wearable accelerometers. Physiol Meas. 2015;36(5):857-72. doi:10.1088/0967-3334/36/5/857

21. Brodie MA, Coppens MJ, Ejupi A, Gschwind YJ, Annegarn J, Schoene D, et al. Comparison between clinical gait and daily-life gait assessments of fall risk in older people. Geriatr Gerontol Int. 2017;17(11):2274-82. doi:10.1111/ggi.12979

22. Xie F, Li S-C, Goeree R, Tarride J-E, O'Reilly D, Lo N-N, et al. Validation of Chinese Western Ontario and McMaster Universities Osteoarthritis Index (WOMAC) in patients scheduled for total knee replacement. Qual Life Res. 2008;17(4):595-601. doi:10.1007/s11136-008-9340-7

23. Bade MJ, Kohrt WM, Stevens-Lapsley JE. Outcomes before and after total knee arthroplasty compared to healthy adults. J Orthop Sports Physl Ther. 2010;40(9):559. doi:10.2519/jospt.2010.3317

24. Yoshida Y, Mizner RL, Ramsey DK, Snyder-Mackler L. Examining outcomes from total knee arthroplasty and the relationship between quadriceps strength and knee function over time. Clin Biomech. 2008;23(3):320-8. doi:10.1016/j.clinbiomech.2007.10.008

25. Podsiadlo D, Richardson S. The timed "Up \& Go": a test of basic functional mobility for frail elderly persons. J Am Geriatr Soc. 1991;39(2):142.

26. Goldman MD, Marrie RA, Cohen JA. Evaluation of the six-minute walk in multiple sclerosis subjects and healthy controls. Multiple Sclerosis. 2008;14(3):383-90.

doi:10.1177/1352458507082607

27. ATS statement: guidelines for the six-minute walk test. Am J Respir Crit Care Med. 2002;166(1):111.

28. Brodie MA, Dean RT, Beijer TR, Canning CG, Smith ST, Menant JC, et al. Symmetry matched auditory cues improve gait steadiness in most people with Parkinson's disease but not in healthy older people. Journal of Parkinson's disease. 2015;5(1):105-16. 29. Hausdorff JM, Rios DA, Edelberg HK. Gait variability and fall risk in communityliving older adults: A 1-year prospective study. Arch Phys Med Rehabil. 2001;82(8):1050-6. 30. Brodie MAD, Psarakis M, Hoang P. Gyroscopic corrections improve wearable sensor data prior to measuring dynamic sway in the gait of people with Multiple Sclerosis. Comput Methods Biomech Biomed Eng. 2016;19(12):1339-46.

31. Bellanca JL, Lowry KA, Vanswearingen JM, Brach JS, Redfern MS. Harmonic ratios: A quantification of step to step symmetry. J Biomech. 2013;46(4):828-31. doi:10.1016/j.jbiomech.2012.12.008

32. Menz HB, Lord SR, Fitzpatrick RC. Acceleration Patterns of the Head and Pelvis When Walking Are Associated With Risk of Falling in Community-Dwelling Older People. 2003. p. M446-M52.

33. Fritz CO, Morris PE, Richler JJ. Effect Size Estimates: Current Use, Calculations, and Interpretation. J Exp Psychol Gen. 2012;141(1):2-18. doi:10.1037/a0024338

34. Coolican H. Research Methods and Statistics in Psychology. Sixth edition. ed:

Psychology Press - M.U.A.; 2014. 
35. Koo S, Andriacchi TP. The knee joint center of rotation is predominantly on the lateral side during normal walking. J Biomech. 2008;41(6):1269-73.

doi:10.1016/j.jbiomech.2008.01.013

36. Kozanek M, Hosseini A, Liu F, Van de Velde SK, Gill TJ, Rubash HE, et al.

Tibiofemoral kinematics and condylar motion during the stance phase of gait. J Biomech. 2009;42(12):1877-84. doi:10.1016/j.jbiomech.2009.05.003

37. Samy DA, Wolfstadt JI, Vaidee I, Backstein DJ. A Retrospective Comparison of a Medial Pivot and Posterior-Stabilized Total Knee Arthroplasty With Respect to PatientReported and Radiographic Outcomes. J Arthroplasty. 2018;33(5):1379-83. doi:10.1016/j.arth.2017.11.049

38. Pritchett JW. Patients Prefer A Bicruciate-Retaining or the Medial Pivot Total Knee Prosthesis. J Arthroplasty. 2011;26(2):224-8. doi:10.1016/j.arth.2010.02.012

39. Warth LC, Ishmael MK, Deckard ER, Ziemba-Davis M, Meneghini RM. Do Medial Pivot Kinematics Correlate With Patient-Reported Outcomes After Total Knee Arthroplasty? J Arthroplasty. 2017;32(8):2411-6. doi:10.1016/j.arth.2017.03.019

40. Ko V, Naylor JM, Harris IA, Crosbie J, Yeo AET. The six-minute walk test is an excellent predictor of functional ambulation after total knee arthroplasty. BMC Musculoskel Disord. 2013;14:145-5.

41. Taniguchi M, Sawano S, Kugo M, Maegawa S, Kawasaki T, Ichihashi N. Physical Activity Promotes Gait Improvement in Patients With Total Knee Arthroplasty. J Arthroplasty. 2016;31(5):984-8. doi:10.1016/j.arth.2015.11.012

42. Yilmaz Yelvar GD, Çirak Y, Dalkilinç M, Demir YP, Baltaci G, Kömürcü M, et al. Impairments of postural stability, core endurance, fall index and functional mobility skills in patients with patello femoral pain syndrome. J Back Musculoskelet Rehabil. 2016. 
Figure legends

Figure 1 The placements of inertia sensors at the head and waist

Figure 2 The postural sway of control and patients with two total knee arthroplasty (TKA) prosthesis designs during the near-tandem stance test

Figure 3 Data collection during 6 minute walk test

Figure 4 Low-pass filtered was used to identify the real transient gait rotations 
Figures

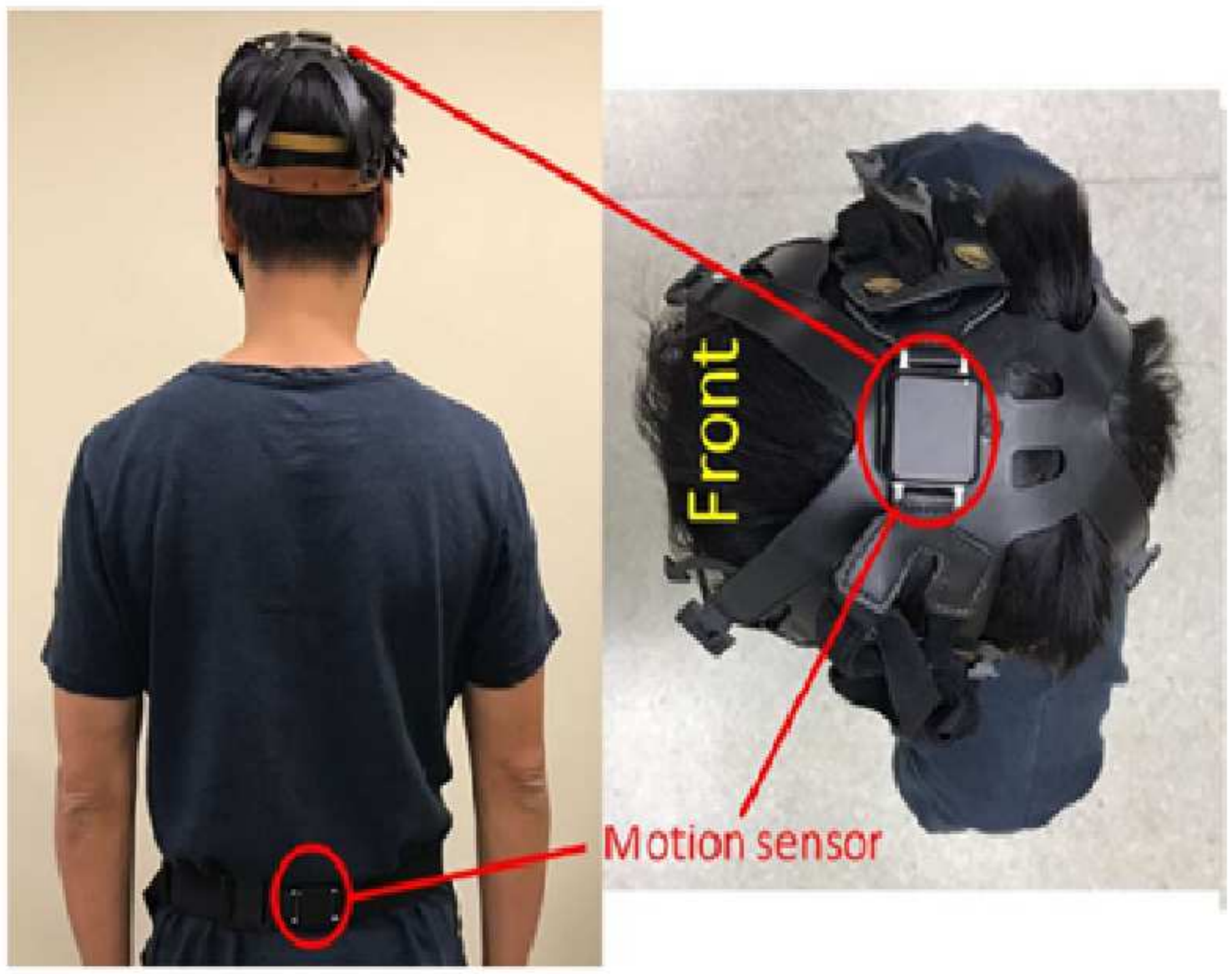

Figure 1

The placements of inertia sensors at the head and waist 

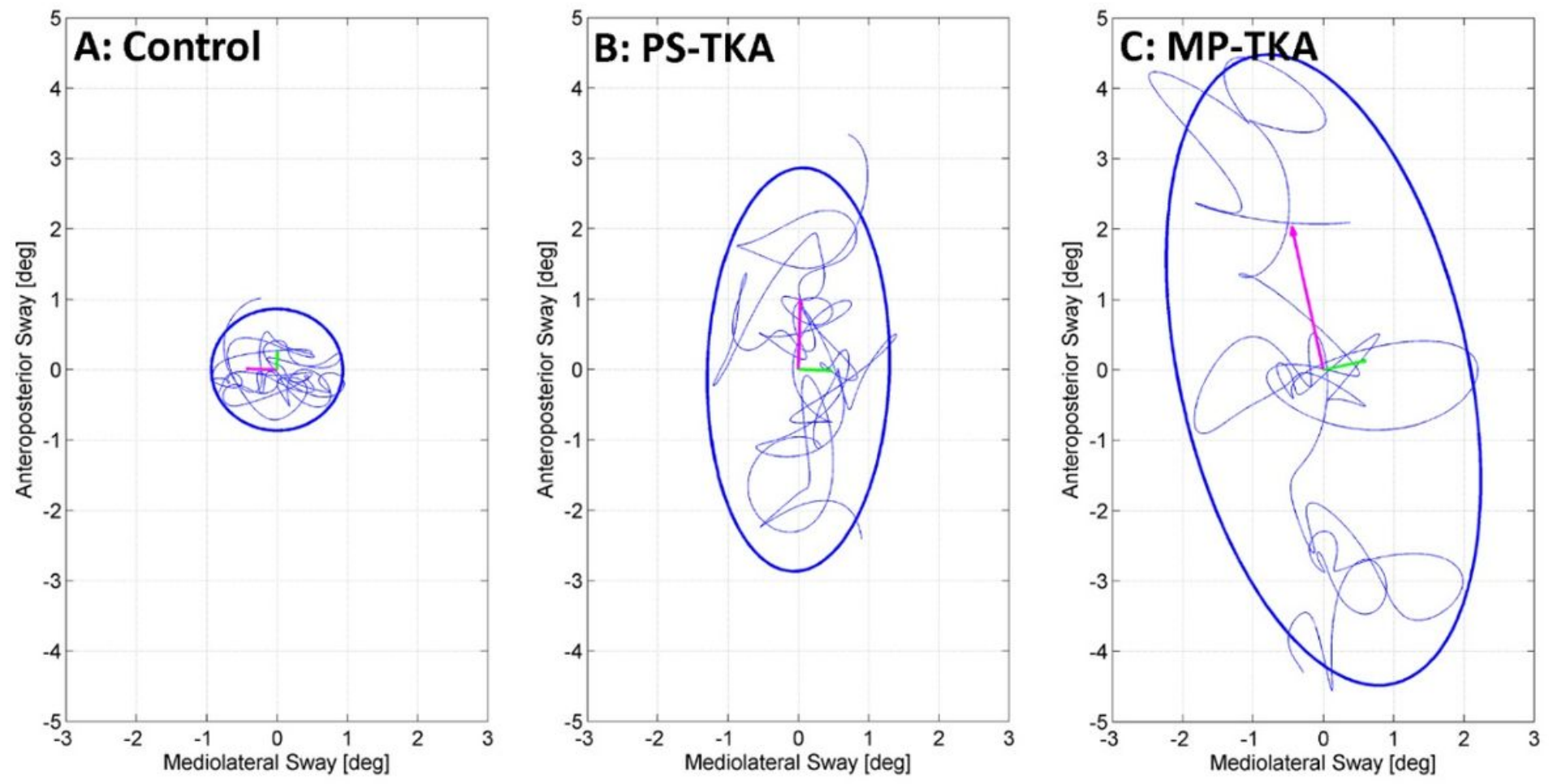

Figure 2

The postural sway of control and patients with two total knee arthroplasty (TKA) prosthesis designs during the near-tandem stance test

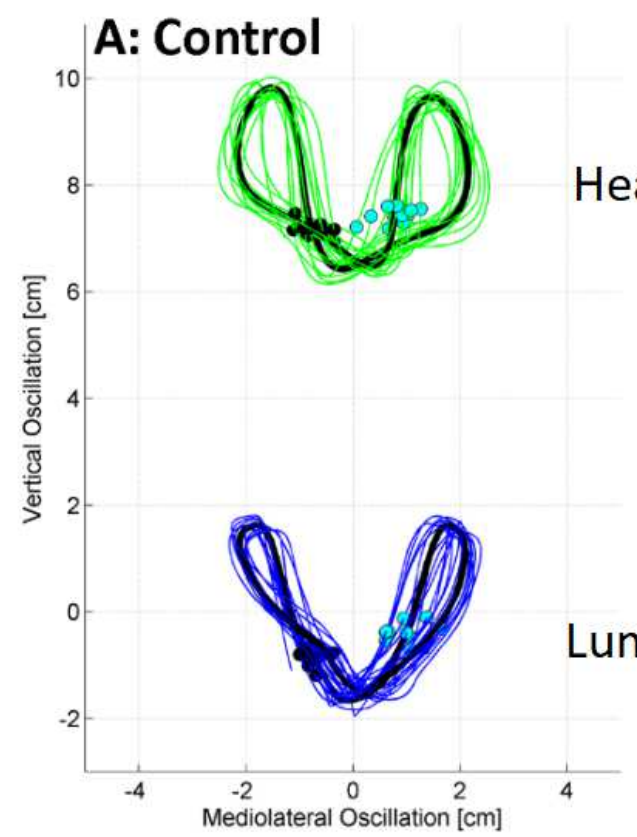

B: PS-TKA

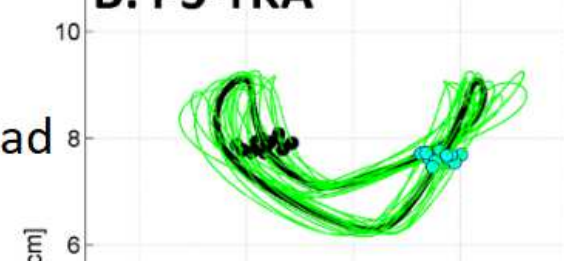

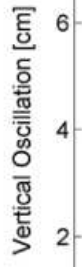

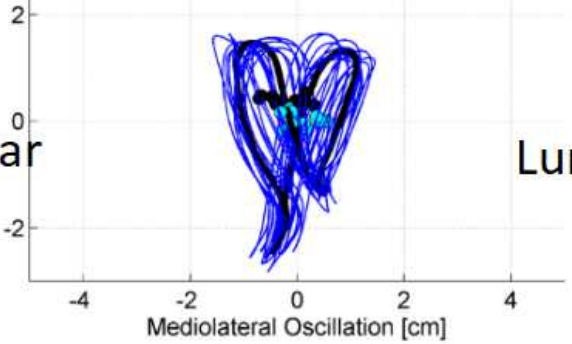

C: MP-TKA

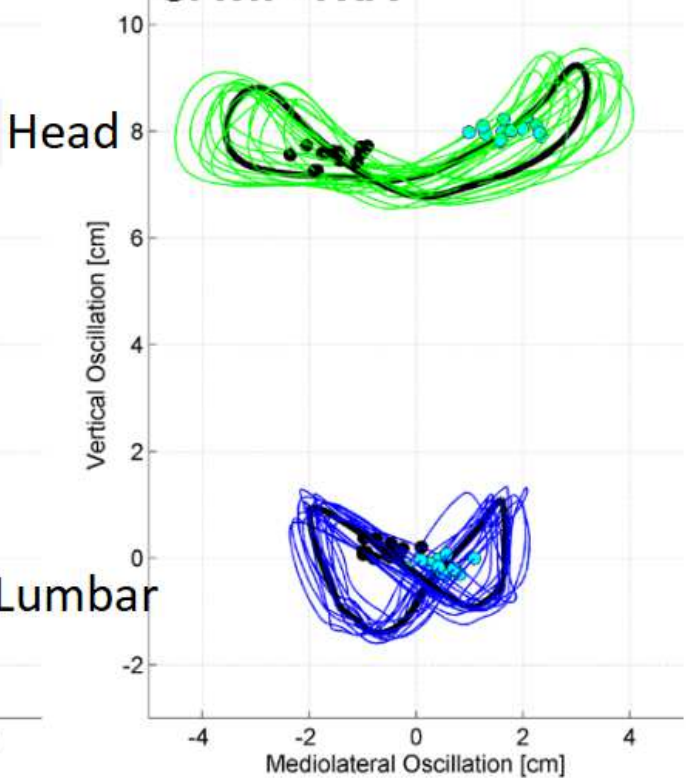

Figure 3 


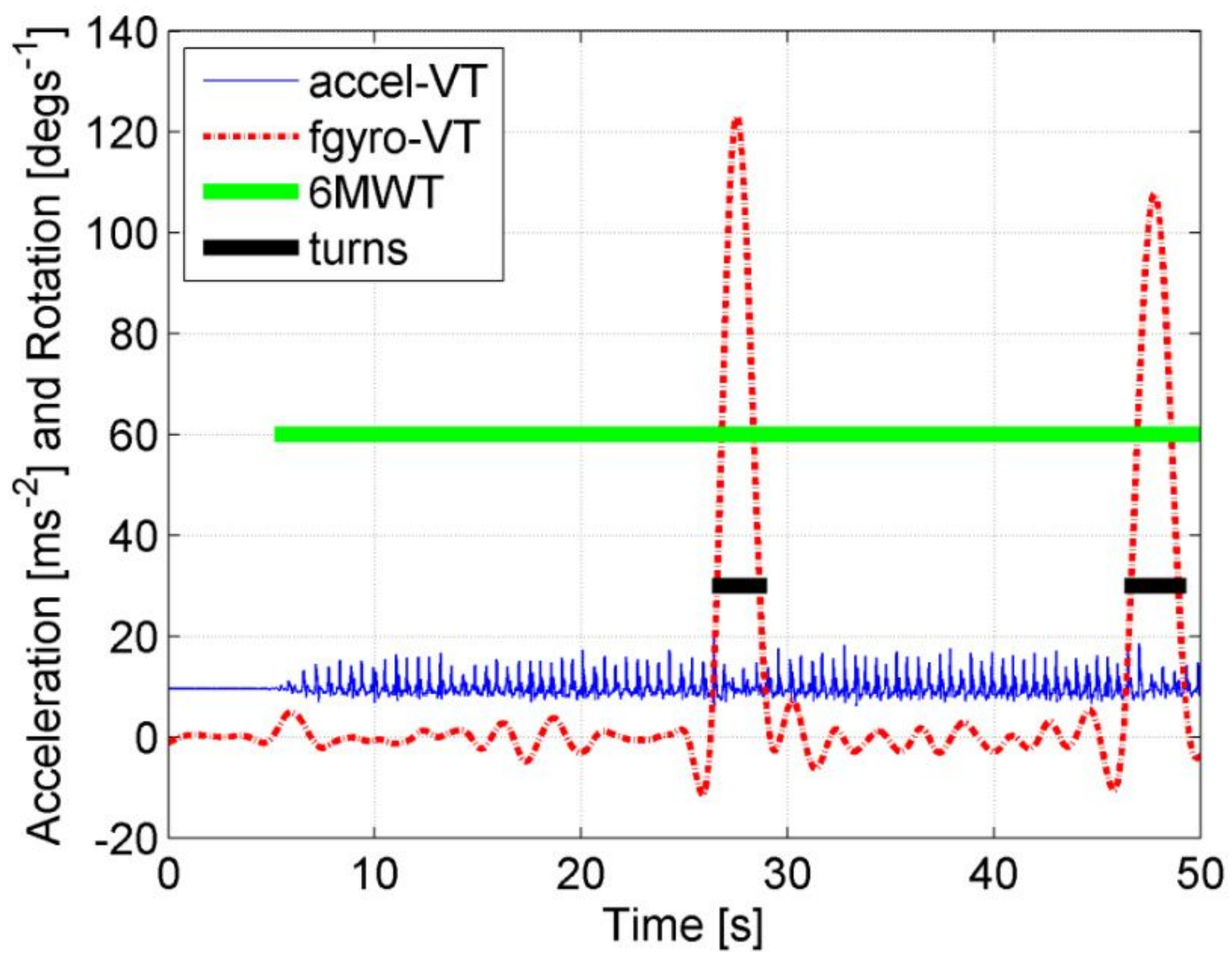

Figure 4

Low-pass filtered was used to identify the real transient gait rotations

\section{Supplementary Files}

This is a list of supplementary files associated with this preprint. Click to download.

- CONSORTchecklist.pdf

- CONSORTabstractchecklist.pdf

- STROBEchecklistcrosssectionalR.pdf

- Equations.pdf 